\title{
Wideband PA Design: The "Continuous" Mode of Operation
}

\author{
Paul. J Tasker, Vince Carrubba, Peter Wright, Jonny Lees, Johannes Benedikt and Steve Cripps \\ Centre for High Frequency Engineering, School of Engineering, Cardiff University, CF24 3AA, \\ Cardiff, Wales, UK \\ tasker@cf.ac.uk
}

\begin{abstract}
The introduction and formulation of the "Continuous" modes of transistor operation has provided an alternative design route for the realization of high efficiency power amplifiers (PA's) over a wide bandwidth (approaching an octave). The formulation of the "Continuous" modes provides a mathematical framework for extending the fundamental/harmonic impedance space over which the power and efficiency performance of the traditional PA modes, i.e. Class, B, Class F, etc, can be maintained. This mathematical framework has been experimentally validated using RF I-V waveform engineering measurements systems and the realization of demonstrator PA prototypes.
\end{abstract}

Index Terms - Microwave measurements, microwave theory and techniques, power amplifiers, power transistors, wideband.

\section{INTRODUCTION}

The overall efficiency of wireless communication networks is predominantly determined by the Power amplifier (PA) stage. Low efficiency generally translates into increased running costs for base stations and reduced battery life for mobile handsets. In narrow band systems it has been possible to utilize "traditional" modes of operation, Class B, Class F, etc, to realize high efficiency PA's. However, these modes require the output matching circuit to present short or open terminations to the relevant harmonics. This termination requirement presents a major circuit design challenge if the application requires wideband power amplifiers. The development of emerging 4G (Fourth Generation) multi-purpose wireless communication networks, such as LTE (Long Term Evolution) that provide higher data-rates (downlink peak rates of at least $100 \mathrm{Mbit} / \mathrm{s}$ and uplink of at least $50 \mathrm{Mbit} / \mathrm{s}$ ) motivates the microwave community to improve PA performance also in terms of bandwidth. Hence, these new communication systems where increasing bandwidth is very important necessitate a new approach to design high efficiency power amplifiers going beyond the "traditional" modes of operation.

In the "traditional" modes of operation, described in the Snider's paper [1], utilized basic waveform building blocks. By avoiding the simultaneous presence of harmonic voltage and current components in their selection high efficiency results. Since, the resulting voltage and current components are harmonically rich, this necessitates the need for harmonic short or open circuit terminations in their practical realization.

On going research activity [2-6] both theoretically and experimentally has indicated that there are alternative modes of operation that can also provide for high efficiency that do allow for the simultaneous, same frequency, presence of harmonic voltage and current components, i.e. Class E [5,6], Class J [3]. The formulation of the "Continuous" modes provides a mathematical framework to describe sets of fundamental/harmonic impedance reactive loci that support specific examples of this mixed mode of operation; delivering the same power and efficiency as the traditional modes on which they are based.

\section{THEORETICAL ANALYSIS}

The power and efficiency performance of Power Amplifiers is mathematically determined by analyzing the dynamic, time varying, voltage $\mathrm{V}(\mathrm{t})$ and current $\mathrm{I}(\mathrm{t})$ waveforms that are present on the Power Amplifiers Transistor Terminals.

$$
V(t)=f(t) \text { and } I(t)=g(t)
$$

Class A, the reference mode of operation, with a theoretical maximum efficiency of $50 \%$, is defined as both the voltage and current waveforms being sinusoidal.

$$
\begin{aligned}
& V(t)=f(t)=V_{D C}(1-\sin (\omega t)) \\
& I(t)=g(t)=\frac{I_{\max }}{2}(1+\sin (\omega t))
\end{aligned}
$$

In this case the waveforms contain no harmonic components. Efficiency performance can be improved via the introduction of harmonic components to modify the shape of the current and/or voltage waveforms. In the work of Snider [1] the current waveform is modified to a half rectified sinusoidal waveform; 


$$
I(t)=g(t)=\frac{I_{\max }}{2}\left(\frac{2}{\pi}+\sin (\omega t)-\frac{4}{\pi} \sum_{r=1}^{\infty} \frac{\cos (2 r \omega t)}{4 r^{2}-1}\right)
$$

The half rectified sinusoidal current waveform, which contains even harmonics, has the same fundamental component as the reference sinusoidal waveform but a reduced $(2 / \pi)$ DC level. This is what is exploited in Class $\mathrm{B}$ operation to provide for an increase in efficiency of $(\pi / 2)$ compared to Class A; theoretical maximum $78.5 \%$. The Class B voltage waveform is unchanged, sinusoidal; hence for the transistor to achieve this mode of operation the even harmonic current components must be terminated by short circuits.

In class $\mathrm{F}$ the voltage wave is modified to become a square waveform.

$$
V(t)=f(t)=V_{D C}\left(1-\frac{4}{\pi} \sum_{r=1}^{\infty} \frac{\sin ((2 r-1) \omega t)}{2 r-1}\right)
$$

The square waveform, which has odd harmonics, has an increased $(4 / \pi)$ fundamental component as the reference sinusoidal waveform with the same DC level. Thus in Class $\mathrm{F}$ the efficiency increases by an additional factor $(4 / \pi)$; theoretical maximum $100 \%$. Note, for the transistor to achieve this mode of operation in addition to the class B short circuit termination condition on the even harmonics current components the odd harmonic voltage components must be terminated by open circuits.

Targeting these modes has, until recently, dominated the high efficient PA amplifier design strategy. However, the short and open circuits harmonic terminations has limited their consideration and suitability for wideband applications.

The recently introduced "continuous" mode concept overcomes this bandwidth concern [7]. It provides for alternative impedance terminating solutions that can provide for the same output power and efficiency performance as their reference traditional mode. These impedance solutions are formulated by multiplication of either the current or voltage reference waveform by the following operator;

$$
h(t)=(1-\alpha \cos (\beta \omega t)-1 \leq \alpha \leq 1
$$

This action will generate a new family of waveforms all having the same DC level and the same fundamental component as the original reference waveform. Hence, a new continuous set of solutions with the same RF output power and efficiency can be identified. A characteristic feature of these new solutions is that they require, or support, a reactive fundamental component and reactive harmonic terminations; hence they are more compatible with broadband circuit behavior than the original reference waveform that necessitates a real fundamental impedance coupled with harmonic short and/or open circuit terminations.

\section{A. Continuous Class B}

Lets consider the utilization of the operator introduced in equation (5) for $\beta=1$ on the Class B voltage waveform. In this case the following set of voltage waveforms solutions are defined when $\alpha$ is varied from -1 to 1 ;

$$
\begin{aligned}
& V(t)=f(t) h(t) \\
& V(t)=V_{D C}(1-\sin (\omega t))(1-\alpha \cos (\omega t)) \\
& V(t)=V_{D C}\left(1-\sin (\omega t)-\alpha \cos (\omega t)+\frac{\alpha}{2} \sin (2 \omega t)\right)
\end{aligned}
$$

Note, all these waveforms have the same DC level and the real fundamental component; hence a theoretical efficiency of $78.5 \%$. The corresponding normalized $V(t) / V_{D C}$ voltage waveforms are shown graphically in figure 1. They highlight that in order to support these modes the transistor must be able to support an increased maximum voltage swing. This, for example, is not an issue for $\mathrm{GaN}$ device technology.

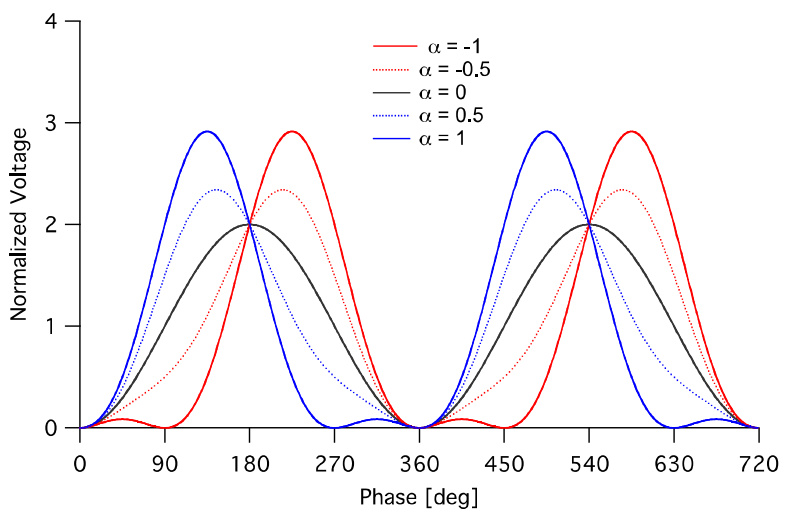

Fig. 1. Family of normalized voltage waveform associated with the continuous class B mode of operation described by equation (6).

Another important observation is that the waveforms computed for $|\alpha|=1$ are those defined in the Class J mode of operation introduced by Cripps [3]. The continuous formulation identifies that Class B and Class $\mathrm{J}$ are alternative solutions of the same mode of operation; hence explaining why they have the same theoretical efficiency and output power prediction. 
The corresponding coupled fundamental and second harmonic load impedance loci as a function of $\alpha$ are given as follows;

$$
\begin{aligned}
& Z_{1}=\frac{2 V_{D C}}{I_{\max }}(1+j \alpha)=R_{o p t}(1+j \alpha) \\
& Z_{2}=\frac{2 V_{D C} 3 \pi}{8 I_{\max }}(-j \alpha)=R_{o p t} \frac{3 \pi}{8}(-j \alpha)
\end{aligned}
$$

Key feature to note is that these impedances are defined at the transistor current generator reference plane, hence are frequency independent. Wideband operation requires the design of an output matching circuit that can track the coupled fundamental/second harmonic reactive impedance variation described by considering $\alpha$ varying as a function of frequency. Alternatively the current waveform rather than voltage waveform could be operated on by $h(t)$ giving another continuous mode solution, defined by loci in the admittance rather than impedance plan in this case.

\section{B. Continuous Class F}

Lets consider the utilization of the operator introduced in equation (5) for $\beta=1$ on the Class F voltage waveform. In this case the following set of voltage waveforms solutions are defined;

$$
\begin{aligned}
& V(t)=f(t) h(t) \\
& V(t)=V_{D C}\left(1-\frac{4}{\pi} \sum_{r=1}^{\infty} \frac{\sin ((2 r-1) \omega t)}{2 r-1}\right)(1-\alpha \cos (\omega t)) \\
& V(t)=V_{D C}\left(\begin{array}{l}
1-\alpha \cos (\omega t)-\frac{4}{\pi} \sum_{r=1}^{\infty} \frac{\sin ((2 r-1) \omega t)}{2 r-1} \\
+\frac{2 \alpha}{\pi} \sum_{r=1}^{\infty} \frac{4 r \sin ((2 r) \omega t)}{4 r^{2}-1}
\end{array}\right)
\end{aligned}
$$

Note, again all these waveforms have the same DC level and the real fundamental component; hence theoretical efficiency of $100 \%$. The corresponding normalized $V(t) / V_{D C}$ voltage waveforms are shown graphically in figure 2. They highlight that again in order to support these modes the transistor must be able to support an increased maximum voltage swing, approaching 4 times the DC rail voltage. This most likely is not an issue for GaN device technology.

The corresponding fundamental and harmonic load impedance requirement are given as follows;

$$
\begin{aligned}
& Z_{1}=\frac{2 V_{D C}}{I_{\max }}\left(\frac{4}{\pi}+j \alpha\right)=R_{o p t} \frac{4}{\pi}\left(1+j \frac{\pi}{4} \alpha\right) \\
& Z_{2 r}=\frac{2 V_{D C}}{I_{\max }}(-j 2 \alpha r)=R_{o p t} \frac{4}{\pi}\left(-j \frac{\pi}{2} \alpha r\right) \\
& Z_{2 r=1}=\infty
\end{aligned}
$$

In this case, while the even harmonic open circuit condition remains unchanged fundamental impedance loci with a variable reactive component can be supported provided the even harmonics are appropriately reactively terminated. Wideband operation requires the design of a more complex output matching circuit that can track the coupled fundamental/even harmonic reactive impedance variation described by considering $\alpha$ varying as a function of frequency. It is envisaged that this can only be achieved over a finite bandwidth. Rather than using the infinite bandwidth square wave, generating a reference waveform based on including only the third harmonic will provide a continuous set of waveforms having a maximum theoretical efficiency to $90.6 \%$ [13]. Again, the current waveform rather than voltage waveform could be operated on by $h(t)$ giving another continuous mode solution, again defined by loci in the admittance rather than impedance plan in this case.

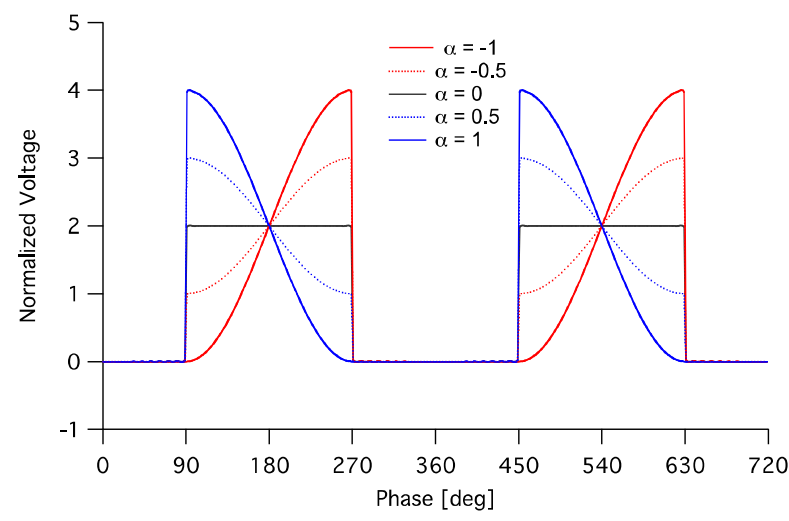

Fig. 2. Family of normalized voltage waveform associated with the continuous class $\mathrm{F}$ mode of operation described by equation (8).

\section{EXPERIMENTAL VALIDATION}

Continuous Class B and band limited Class F operation have been experimentally verified by performing RF I-V waveform measurements [8] while varying the fundamental and second harmonic load along the computed coupled fundamental/second harmonic variable reactive impedance loci [7, 9-13]. 


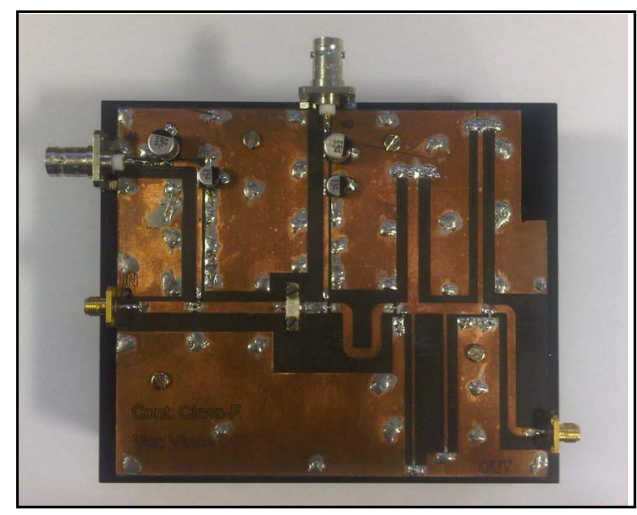

Fig. 3. Prototype wideband high efficiency PA using a GaN $10 \mathrm{~W}$ transistor based on the continuous Class-F mode of operation [13].

\section{PA PROTOTYPING}

Using these experimentally determined coupled fundamental/second harmonic impedance loci output matching circuits have been designed to track these over bandwidth's approach one octave. These resulting realized PA prototypes have demonstrated high efficiency operation over a very wide bandwidth $[9,12,13]$. For example, the prototype PA shown in figure 3 exploited the continuous Class $\mathrm{F}$ solution to demonstrate efficiencies of greater than $75 \%$ over a frequency range from $0.55 \mathrm{GHz}$ to $0.9 \mathrm{GHz}$ [13].

\section{SUMMARY}

The developed formulation of the "continuous" modes provides a mathematical framework for extending the fundamental/harmonic impedance space over which the power and efficiency performance of the traditional PA modes, i.e/ Class B, Class F, etc. can be maintained. Designing output matching circuits that track these fundamental/second harmonic reactive impedance loci over bandwidths approach an octave has proved feasible enabling the realization of wideband high efficiency prototype PA's.

\section{ACKNOWLEDGMENT}

The authors would like to acknowledge the support of Cree for supplying GaN transistors for the experimental validation and PA prototyping.

\section{REFERENCES}

[1] Snider, D.M., "A theoretical analysis and experimental confirmation of the optimally loaded and overdriven R.F. power amplifier", I.E.E.E. Trans. Electron. Dev., Vol. 14, no. 12, Dec. 1967, pp 851-857.

[2] Rhodes, J.D., "Output universality in maximum efficiency linear power amplifiers", Intl. Jour. Circuit Theory and Appl., Vol 31, 2003, pp385-405.

[3] Cripps, S.C., "RF Power amplifiers for Wireless Communications", 2nd Edition, Artech House Publishers, 2006.

[4] Raab, F., "Class-F Power amplifiers with Maximally Flat Waveforms", I.E.E.E. Trans. MTT., Vol. 31, no. 11, Nov. 1997, pp 2007-012.

[5] Raab, F., "Idealised operation of the Class E tuned amplifier", I.E.E.E Trans. Circuits and Sys., Vol.24, No. 12, Dec 1977, pp 725-735.

[6] Sokal, N.O.; Sokal A.D., "Class E-A New Single-Ended Class of High-Efficiency Tuned Switching Power amplifiers", I.E.E.E. Journal of Solid-State Circuits, Vol. 10, no. 3, June 1975, pp 168-176.

[7] Cripps, S.C.; Tasker, P.J.; Clarke A.L.; J.Lees, J.; Benedikt, J.; "A New General Formulation for High Efficiency R.F. Amplifiers.", IEEE Microwave and Wireless Components Letters, Oct. 2009.

[8] Tasker, P.J.; "Practical waveform engineering. Microwave Magazine”, IEEE •Volume: 10 , Issue: 7, 2009 , Page(s): 65 $-76$.

[9] PA. Wright, P.; Lees, J.; Benedikt, J.; Tasker, P.J.; Cripps, S.C.; "A Methodology for Realizing High Efficiency Class$\mathrm{J}$ in a Linear and Broadband", -Microwave Theory and Techniques, IEEE Transactions on •Volume: 57, Issue: 12 , Part: 2 Digital Object Identifier: 10.1109/TMTT.2009.2033295 •Publication Year: 2009 , Page(s): 3196 - 3204.

[10] Carrubba, V.; Clarke, A. L.; Akmal, M.; Lees, J.; Benedikt, J.; Tasker, P. J.; Cripps, S. C.; "The Continuous Class-F Mode Power Amplifier", Microwave Conference (EuMC), 2010 European, 2010 , Page(s): 1674 - 1677.

[11] Carrubba, V.; Clarke, A.L.; Akmal, M.; Lees, J.; Benedikt, J.; Tasker, P.J.; Cripps, S.C.; "On the Extension of the Continuous Class-F Mode Power Amplifier", Microwave Theory and Techniques, IEEE Transactions on, Volume: 59, Issue: 5, 2011 , Page(s): $1294-1303$.

[12] Powell, J. R.; Uren, M. J.; Martin, T.; McLachlan, A.; Tasker, P. J.; Bell, J. J.; Saini, R. S.; Woodington, S. P.; Benedikt, J.; Cripps, S. C.; "GaAs X-band high efficiency (65\%) broadband $(30 \%)$ amplifier MMIC based on the Class B to Class J continuum", Microwave Symposium Digest (MTT), 2011 IEEE MTT-S International, June 2011.

[13] Carrubba, V.; Lees, J.; Benedikt, J.; Tasker, P. J.; Cripps, S. C.; "A novel highly efficient broadband continuous class-F RFPA delivering $74 \%$ average efficiency for an octave bandwidth", Microwave Symposium Digest (MTT), 2011 IEEE MTT-S International, June 2011. 